\title{
Lack of prognostic significance for major adverse cardiac events of soluble suppression of tumorigenicity 2 levels in patients with ST-segment elevation myocardial infarction
}

\author{
Min Kim ${ }^{1}$, Dae In Lee ${ }^{1}$, Ju-Hee Lee ${ }^{1}$, Sang Min Kim ${ }^{1}$, Sang Yeub Lee ${ }^{1,2}$, \\ Kyung-Kuk Hwang, ${ }^{1,2}$, Dong-Woon Kim ${ }^{1,2}$, Myeong-Chan Cho ${ }^{1,2}$, Jang-Whan Bae ${ }^{1,2}$ \\ ${ }^{1}$ Regional Cardiovascular Center, Division of Cardiology, Department of Internal Medicine, \\ Chungbuk National University Hospital, Cheongju, Republic of Korea \\ ${ }^{2}$ Department of Internal Medicine, College of Medicine, \\ Chungbuk National University, Cheongju, Republic of Korea
}

\begin{abstract}
Background: Elevation of soluble suppression of tumorigenicity 2 (sST2) is associated with cardiac fibrosis and hypertrophy. Under investigation herein, was whether sST2 level is associated with major adverse cardiac events (MACE) and left ventricular $(L V)$ remodeling after primary percutaneous coronary intervention (PCI) in patients with acute ST-segment elevation myocardial infarction (STEMI). Methods: In total, this study included 184 patients who underwent successful primary PCI. A subsequent guideline-based medical follow-up was included (61.4 \pm 11.8 years old, $85 \%$ male, $21 \%$ with Killip class $\geq I$ ). sST2 concentration correlations with echocardiographic, angiographic, laboratory parameters, and clinical outcomes in STEMI patients were evaluated.

Results: The median sST2 level was $60.3 \mathrm{ng} / \mathrm{mL} ; 6$ (3.2\%) deaths occurred within 1 year. The sST2 level correlated with LV ejection fraction (LVEF) changes from baseline to 6 months ( $r=-0.273$; $p=0.006)$ after adjustment for echocardiographic parameters including wall motions score index (WMSI). Recovery of LVEF at 6 months was highest in the tertile 1 group ( $\triangle 6$ months - baseline LVEF; tertile 1, $p=0.001$; tertile 2, $p=0.319$; tertile $3, p=0.205$ ). The decrease in WMSI at 6 months was greater in the tertiles 1 and 2 groups than in the tertile 3 group ( $\Delta 6$ months - baseline WMSI; tertile 1 , $p=0.001$; tertile $2, p=0.013$; tertile $3, p=0.055$ ). There was no association between sST2 levels and short-term (log rank $p=0.598)$ and long-term $(p=0.596)$ MACE.

Conclusions: sST2 concentration have predictive value for $L V$ remodeling on echocardiography in patients with STEMI who underwent primary PCI. However, sST2 concentration was not associated with short-term and long-term MACE. (Cardiol J 2021; 28, 2: 244-254)
\end{abstract}

Key words: suppression of tumorigenicity 2 protein, myocardial infarction, left ventricular remodeling

\section{Introduction}

Acute ST-segment elevation myocardial infarction (STEMI) makes a significant contribution to morbidity and mortality in many parts of the world [1-4]. It is well known that early diagnosis and proper management, especially delay from the on- set of symptoms to revascularization are important for long-term prognosis [5, 6]. Timely diagnosis allows physicians to stratify their patients by risk, and consequently provides them with the opportunity to select appropriate treatments. Biomarkers have been used to assist with timely diagnosis and to predict precise short- or long-term prognosis in

Address for correspondence: Jang-Whan Bae, MD, PhD, 776, 1 Sunwhan-ro, Seowon-gu, Cheongju-si, Chungcheongbuk-do, Republic of Korea, 28627, tel: +82-43-269-6707, fax: +82-43-273-3252, e-mail: drcorazon@hanmail.net

Received: $16.01 .2020 \quad$ Accepted: 11.02 .2020

This article is available in open access under Creative Common Attribution-Non-Commercial-No Derivatives 4.0 International (CC BY-NC-ND 4.0) license, allowing to download articles and share them with others as long as they credit the authors and the publisher, but without permission to change them in any way or use them commercially. 


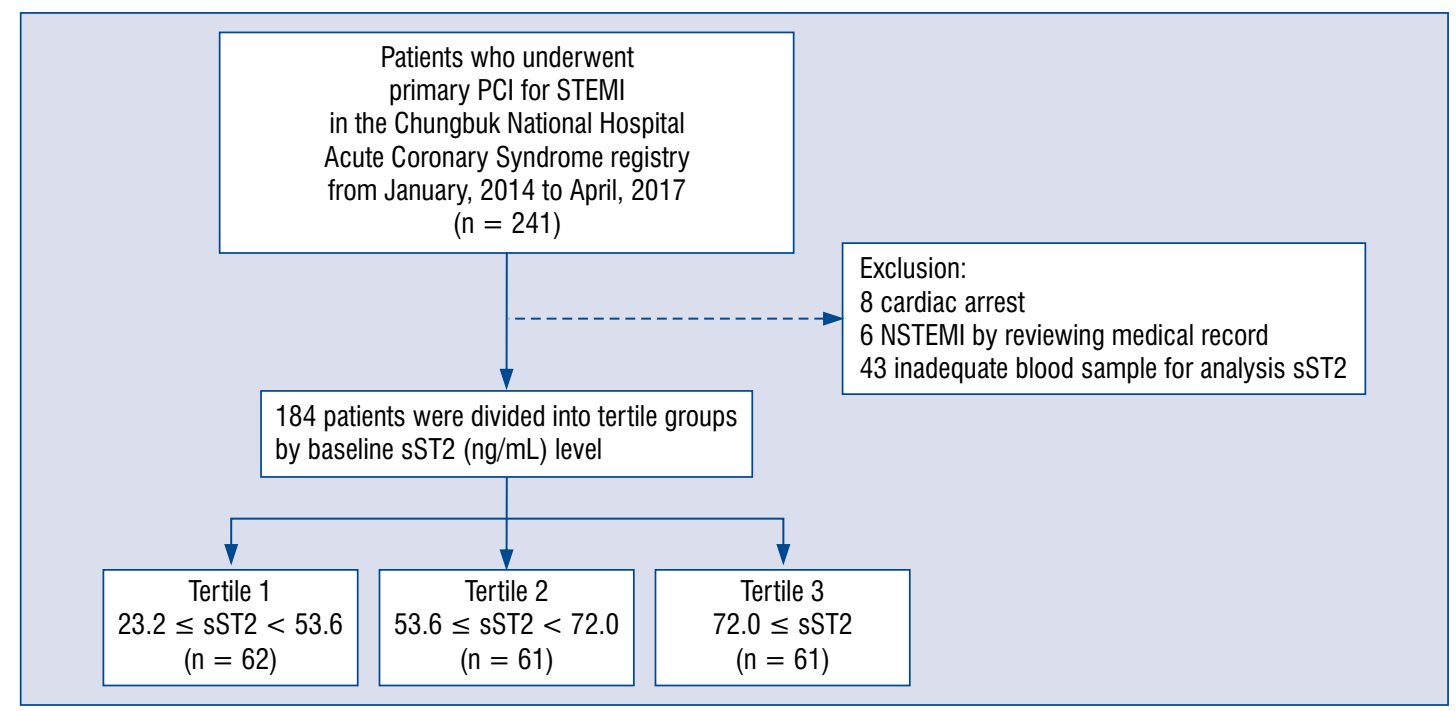

Figure 1. Study flow chart of patient enrolment; NSTEMI — non-ST-segment elevation myocardial infarction; STEMI — ST-segment elevation myocardial infarction; $\mathrm{PCl}$ - percutaneous coronary intervention.

STEMI patients. As a result, cardiac biomarkers, such as creatine phosphokinase (CPK), creatinine kinase-myocardial band (CK-MB), cardiac specific troponins, and natriuretic peptides, are widely used to diagnose and predict prognosis in patients with STEMI [7-9]. Circulating soluble suppression of tumorigenicity 2 (sST2) is a known biomarker of cardiac remodeling and inflammation, especially in heart failure (HF) patients. It is thought to act as a decoy receptor for interleukin-33, rendering it unavailable to membrane-bound ST2 receptors that medicate anti-inflammatory and anti-fibrotic effects [10-12]. Several studies have reported that increased SST2 in the initial phase of STEMI is closely related to adverse outcomes, both in the short- and long-term [13-16]. However, current guidelines do not recommend the examination of SST2 as a biomarker in the treatment for STEMI. Therefore, under investigation herein, are the associations between the concentration of sST2 and the clinical and echocardiographic outcome. Its performance was compared to established risk predictors such as the Killip classification, Thrombolysis in Myocardial Infarction (TIMI) risk score, and the Canadian acute coronary syndrome (CACS) score.

\section{Methods}

\section{Study design and population}

The study was a single center, retrospective, observational study. The study population consisted of 184 patients who underwent successful primary percutaneous coronary intervention $(\mathrm{PCI})$ for STEMI from January 2014 to April 2017 at the Chungbuk National University Hospital, Republic of Korea. In total, 184 patients were included. Figure 1 shows the study flow chart. Written informed consent was obtained from all patients, and exclusion criteria were as follows: (1) end-stage renal disease requiring dialysis; (2) life expectancy $<12$ months; (3) pre-hospital or pre-PCI cardiac arrest; (4) prior coronary artery bypass graft surgery; (5) known malignancy or inflammatory disease. The study complied with the Declaration of Helsinki and was approved by the institutional review board (IRB) of Chungbuk National University Hospital (CBNUH 2018-07-013).

\section{Laboratory assays}

All plasma samples were collected before primary PCI with arterial access. The plasma samples were stored in plastic cryovials at $-80^{\circ} \mathrm{C}$ at the Chungnbuk National University Hospital Brach Bank of the Korean Biobank Network until required for analysis. The sST2 concentration in blood specimens was measured using an enzymelinked immunosorbent assay kit (ELISA) (Elabscience Biotechnology, China) [17]; calibration and standardization were performed according to the manufacturer instructions. Intra-assay and interassay coefficients of variance were reported as $<2.5 \%$ and $<4.0 \%$, respectively [18]. To examine a dose-response relationship between sST2 and outcomes, tertiles of sST2 were analyzed and defined as tertile $1: 0<53.6 \mathrm{ng} / \mathrm{mL}$, tertile $2: 53.6 \leq \mathrm{sST} 2$ $<72.0(\mathrm{ng} / \mathrm{mL})$, and tertile $3: \mathrm{sST} 2 \geq 72.0(\mathrm{ng} / \mathrm{mL})$. 


\section{Initial treatment strategies}

The initial treatments in hospitalized patients with STEMI were administration of loading doses for dual antiplatelet agents and primary PCI that was performed after intravenous administration of 7,000 IU of heparin. Second generation drugeluting stents were implanted in all patients, and the decision on whether to use intravascular imaging modalities, an intra-aortic balloon pump, thrombectomy devices, or extracorporeal membrane oxygenation devices was made by the operator.

Time for revascularization was determined in three ways: (1) time from symptom onset to balloon inflation, (2) time from symptom onset to medical contact, and (3) time from medical contact to balloon inflation. All patients received standard medical treatment with revascularization at the discretion of the attending physician.

\section{Echocardiographic measurement}

All patients underwent transthoracic echocardiography (IE33, Philips Medical System, Andover, MA, USA; Vivid 7, GE Vingmed Ultrasound, Horten, Norway; SC2000, Siemens, Erlangen, Germany) within 12 hours of the index procedure. The left ventricular (LV) systolic function (LV ejection fraction [LVEF]), LV internal dimension at diastole (LVIDd), ratio of the early diastolic peak mitral inflow velocity to early diastolic mitral annular velocity (E/E'), left atrial volume index (LAVI), and wall motion score index (WMSI) were obtained according to the American Society of Echocardiography guidelines [19]. Follow-up echocardiography was performed 6 months after discharge at outpatient clinics. $\triangle \mathrm{LVEF}, \Delta \mathrm{LVIDd}, \Delta \mathrm{E} / \mathrm{E}^{\prime}$, $\Delta \mathrm{LAVI}$, and $\Delta \mathrm{WMSI}$ were defined by subtracting the baseline echocardiographic parameters from the echocardiographic performed 6 months after discharge from initial hospitalization.

\section{Follow-up and endpoint}

Standard medications, including dual antiplatelet agents, beta-blockers, renin-angiotensin-aldosterone system inhibitors, statins, and nitrates, were provided by responsible physicians according to the guidelines. The primary endpoint was major adverse cardiac event (MACE) at 1 year; this comprised of occurrence of cardiovascular death, non-fatal myocardial infarction (MI), and non-fatal stroke. The secondary endpoint was differences in echocardiographic parameters indicating $\mathrm{LV}$ remodeling between baseline and 6 -month followup. The endpoints were obtained by reviewing electronic hospital medical records.

\section{Statistical analysis}

Demographic, clinical, echocardiographic, and laboratory variables were described as means and standard deviation (SD) in normally distributed variables, and variables with a non-normal distribution were described as medians and interquartile range (IQR). The analysis of variance was used to compare normally distributed variables, and the Kruskal-Wallis test was used to compare continuous variables in a state of non-normality. Categorical variables were compared using the $\chi^{2}$ test or the Fisher exact test. Univariable Pearson and Spearman correlation and partial correlation were used to evaluate the magnitude and significance of relationships among continuous variables.

The Wilcoxon signed rank test was used to compare changes in echocardiographic parameters by time within groups. Multiple Cox proportional hazard analyses were performed in an effort to identify independent predictors of 1-year MACE after primary PCI. Variables were retained and entered into a multivariable model if their univariable $\mathrm{p}$ value was $<0.05$.

The Kaplan-Meier method was used to estimate event-free survival, and differences between the curves were compared using the log-rank test.

Analyses were performed using SPSS 25.0 (SPSS Inc, Chicago, IL, USA), and SAS 9.4 (SAS Institute Inc, Cary, NC, USA). P-values (two-tailed) $<0.05$ were considered to indicate statistical significance.

\section{Results}

\section{Patient characteristics related to sST2 tertile}

In total, 184 subjects, who were followed up 1 year after successful primary PCI for STEMI, were evaluated in this study. The mean age of the subjects was $61.4 \pm 11.8$ years, and $15 \%$ were female. In addition, $57 \%$ had a culprit lesion in the left anterior descending artery, the median (IQR) symptom to door time was $120(53,267) \mathrm{min}$, the door to balloon time was $39(30,50) \mathrm{min}, 21 \%$ were Killip classification $\geq 2,54 \%$ were TIMI risk score $>4,17 \%$ were CACS score $>1$, and $100 \%$ presented with STEMI. The median sST2 concentration was $60.3 \mathrm{ng} / \mathrm{mL}\left(25^{\text {th }}, 75^{\text {th }}\right.$ percentile: $48.7,77.3 \mathrm{ng} /$ /mL, respectively; range: $23.2-197.5 \mathrm{ng} / \mathrm{mL}$ ). Of these, $62(33.6 \%)$ patients were included in tertile $1,61(33.2 \%)$ patients were included in tertile 2 , and $61(33.2 \%)$ patients were included in tertile 3. The baseline characteristics of patients stratified by sST 2 concentration are shown in Table 1. Higher sST2 concentration showed an association 
Table 1. Baseline characteristics according to SST2 tertile in patients with ST-segment elevation myocardial infarction.

\begin{tabular}{|c|c|c|c|c|c|}
\hline \multirow[t]{2}{*}{ Variables } & \multirow{2}{*}{$\begin{array}{c}\text { Overall } \\
(n=184)\end{array}$} & \multicolumn{3}{|c|}{ sST2 [ng/mL] } & \multirow[t]{2}{*}{$\mathbf{P}$} \\
\hline & & $\begin{array}{c}\text { Tertile } 1 \\
23.2 \leq \text { sST2 }<53.6 \\
\text { (n = 62) }\end{array}$ & $\begin{array}{c}\text { Tertile } 2 \\
53.6 \leq \mathrm{sST} 2<72.0 \\
\text { (n = 61) }\end{array}$ & $\begin{array}{c}\text { Tertile } 3 \\
72.0 \leq \mathrm{sST} 2 \\
(\mathrm{n}=61)\end{array}$ & \\
\hline Age [years] & $61.4 \pm 11.8$ & $58.4 \pm 9.8$ & $63.0 \pm 12.7$ & $62.5 \pm 12.3$ & 0.058 \\
\hline Body weight [kg] & $67.8 \pm 12.4$ & $68.8 \pm 11.8$ & $66.6 \pm 13.0$ & $68.5 \pm 12.2$ & 0.554 \\
\hline Female & $28(15 \%)$ & $9(15 \%)$ & $8(13 \%)$ & $11(18 \%)$ & 0.763 \\
\hline Baseline HR [bpm] & $76 \pm 20$ & $75 \pm 20$ & $76 \pm 18$ & $77 \pm 22$ & 0.910 \\
\hline Baseline SBP [mmHg] & $130(110,145)$ & $130(110,149)$ & $130(110,140)$ & $130(109,146)$ & 0.885 \\
\hline Baseline DBP [mmHg] & $80(70,90)$ & $80(70,90)$ & $79(70,90)$ & $80(70,90)$ & 0.597 \\
\hline Symptom to door time [min] & $120(53,267)$ & $117(40,280)$ & $120(58,201)$ & $120(49,342)$ & 0.876 \\
\hline Symptom to balloon time [min] & $160(88,300)$ & $162(87,315)$ & $168(87,248)$ & $160(93,380)$ & 0.911 \\
\hline Door to balloon time [min] & $39(30,50)$ & $40(30,52)$ & $37(28,50)$ & $40(33,50)$ & 0.343 \\
\hline Prior angina & $8(4 \%)$ & $3(5 \%)$ & $2(3 \%)$ & $3(5 \%)$ & 1.000 \\
\hline Smoking & $127(69.0 \%)$ & $39(63 \%)$ & $44(72 \%)$ & $44(72 \%)$ & 0.462 \\
\hline Hypertension & $101(55 \%)$ & $28(45 \%)$ & $34(56 \%)$ & $39(64 \%)$ & 0.111 \\
\hline Diabetes & $59(32 \%)$ & $17(28 \%)$ & $18(30 \%)$ & $24(39 \%)$ & 0.332 \\
\hline Culprit lesion: & & & & & 0.660 \\
\hline LAD & $105(57 \%)$ & $37(60 \%)$ & $38(62 \%)$ & $30(49 \%)$ & \\
\hline LCX & $20(11 \%)$ & $6(10 \%)$ & $6(10 \%)$ & $8(13 \%)$ & \\
\hline $\mathrm{RCA}$ & $59(32 \%)$ & $19(31 \%)$ & $17(28 \%)$ & $23(38 \%)$ & \\
\hline Killip class $>$ I & $38(21 \%)$ & $10(16 \%)$ & $10(16 \%)$ & $18(30 \%)$ & 0.125 \\
\hline TIMI risk score $>4$ & $99(54 \%)$ & $27(44 \%)$ & $35(57 \%)$ & $37(61 \%)$ & 0.131 \\
\hline CACS risk score $>1$ & $25(17 \%)$ & $7(11 \%)$ & $11(18 \%)$ & $14(23 \%)$ & 0.226 \\
\hline \multicolumn{6}{|l|}{ Medication: } \\
\hline ASA & $184(100 \%)$ & $62(100 \%)$ & $61(100 \%)$ & $61(100 \%)$ & 1.000 \\
\hline P2Y12 inhibitors* & 175 (95\%) & $60(97 \%)$ & $56(92 \%)$ & $59(97 \%)$ & 0.474 \\
\hline Beta-blocker & $159(86 \%)$ & $56(90 \%)$ & $49(80 \%)$ & $54(89 \%)$ & 0.221 \\
\hline ACEI or ARB & $149(81 \%)$ & $51(82 \%)$ & $50(82 \%)$ & $48(79 \%)$ & 0.892 \\
\hline Statin & $170(93 \%)$ & $58(94 \%)$ & $56(92 \%)$ & $56(93 \%)$ & 0.939 \\
\hline \multicolumn{6}{|l|}{ Laboratory findings: } \\
\hline Initial CPK [IU/L] & $132(85,256)$ & $135(89,259)$ & $130(85,256)$ & $129(78,258)$ & 0.937 \\
\hline Peak CPK [IU/L] & $1895(769,3757)$ & $1594(602,3882)$ & $1888(684,3713)$ & $1927(905,3846)$ & 0.657 \\
\hline Initial CK-MB [ng/mL] & $3.3(1.8,10.9)$ & $2.7(1.7,7.2)$ & $3.2(1.7,10.9)$ & $3.7(1.8,14.8)$ & 0.661 \\
\hline Peak CK-MB [ng/mL] & $\begin{array}{c}184.8 \\
(62.3,300.0)\end{array}$ & $\begin{array}{c}157.4 \\
(60.0,300.0)\end{array}$ & $\begin{array}{c}190.5 \\
(61.0,300.0)\end{array}$ & $\begin{array}{c}188.5 \\
(66.7,300.0)\end{array}$ & 0.719 \\
\hline Peak CK-MB > 300 & $62(34 \%)$ & $20(32 \%)$ & $21(34 \%)$ & $21(35 \%)$ & 0.942 \\
\hline Initial troponin-T [ng/mL] & $0.03(0.01,0.13)$ & $0.02(0.01,0.07)$ & $0.02(0.01,0.11)$ & $0.04(0.01,0.21)$ & 0.273 \\
\hline Peak troponin-T [ng/mL] & $2.74(0.96,6.01)$ & $1.42(0.44,5.89)$ & $3.77(1.12,6.75)$ & $2.92(0.76,5.89)$ & 0.117 \\
\hline Peak troponin-T > 10 & $23(13 \%)$ & $7(11 \%)$ & $10(16 \%)$ & $6(10 \%)$ & 0.569 \\
\hline Initial pro-BNP [pg/mL] $†$ & $\begin{array}{c}90.4 \\
(33.6,394.8)\end{array}$ & $\begin{array}{c}57.5 \\
(24.9,212.0)\end{array}$ & $\begin{array}{c}59.9 \\
(17.9,335.6)\end{array}$ & $\begin{array}{c}172.4 \\
(36.8,926.9)\end{array}$ & 0.339 \\
\hline Initial hs-CRP [mg/L] & $0.16(0.10,0.29)$ & $0.16(0.10,0.29)$ & $0.16(0.11,0.41)$ & $0.17(0.11,0.27)$ & 0.728 \\
\hline Initial WBC [/uL] & $\begin{array}{c}11290 \\
(8830,13700)\end{array}$ & $\begin{array}{c}11065 \\
(8618,14090)\end{array}$ & $\begin{array}{c}11500 \\
(9065,13090)\end{array}$ & $\begin{array}{c}10630 \\
(9060,13695)\end{array}$ & 0.825 \\
\hline Initial eosinophil [/uL] & $11(4,20)$ & $13(4,24)$ & $10(4,20)$ & $10(3,20)$ & 0.524 \\
\hline
\end{tabular}


Table 1 (cont.). Baseline characteristics according to SST2 tertile in patients with ST-segment elevation myocardial infarction.

\begin{tabular}{|c|c|c|c|c|c|}
\hline \multirow[t]{2}{*}{ Variables } & \multirow{2}{*}{$\begin{array}{c}\text { Overall } \\
(n=184)\end{array}$} & \multicolumn{3}{|c|}{ sST2 [ng/mL] } & \multirow[t]{2}{*}{$\mathbf{P}$} \\
\hline & & $\begin{array}{c}\text { Tertile } 1 \\
23.2 \leq \text { sST2 }<53.6 \\
\text { (n = 62) }\end{array}$ & $\begin{array}{c}\text { Tertile } 2 \\
53.6 \leq \mathrm{sST} 2<72.0 \\
(\mathrm{n}=61)\end{array}$ & $\begin{array}{c}\text { Tertile } 3 \\
72.0 \leq \mathrm{sST} 2 \\
(\mathrm{n}=61)\end{array}$ & \\
\hline \multicolumn{6}{|c|}{ Major cardiac event (30 days): } \\
\hline Cardiac death & $6(3 \%)$ & $2(3 \%)$ & $3(5 \%)$ & $1(2 \%)$ & 0.702 \\
\hline Heart failure & $0(0 \%)$ & $0(0 \%)$ & $0(0 \%)$ & $0(0 \%)$ & 1.000 \\
\hline \multicolumn{6}{|c|}{ Major cardiac event (1 year): } \\
\hline Cardiac death & $6(3 \%)$ & $2(3 \%)$ & $3(5 \%)$ & $1(2 \%)$ & 0.702 \\
\hline Heart failure & $0(0 \%)$ & $0(0 \%)$ & $0(0 \%)$ & $0(0 \%)$ & 1.000 \\
\hline
\end{tabular}

*Ticagrelor $124(71 \%)$, prasugrel $17(10 \%)$, clopidogrel $39(19 \%)$ tpro-BNP level was obtained in 19 subjects

Data are presented as number (\%) and mean (standard deviation) or median (interquartile).

Non-parametric continuous variables, which were evaluated by the Kolmogorov-Smimov method, were analyzed by the Kruskal-Wallis test. SST2 - soluble suppression of tumorigenicity 2; HR — heart rate; SBP — systolic blood pressure; DBP — diastolic blood pressure; LAD — left anterior descending artery; LCX — left circumflex artery; RCA — right coronary artery; TIMI - Thrombolysis in Myocardial Infarction;

CACS - Canadian acute coronary syndrome; ASA - acetylsalicylic acid; ACEI — angiotensin-converting enzyme inhibitor; ARB - angiotensin receptor blocker; CPK — creatine phosphokinase; CK-MB — creatine kinase myocardial bound; BNP — B-type natriuretic peptide; hs-CRP high sensitivity C-reactive protein; WBC - white blood cell

with trends for old age, hypertension, higher Killip classification, TIMI risk score, and CACS score, although these were not statistically significant. Furthermore, the sST2 level was not associated with age, body weight, sex, smoking, prior angina, diabetes, culprit lesion, and time from symptom onset to initiation of primary PCI. The levels of initial and peak CPK, CK-MB, and cardiac specific troponin were not significantly higher in the higher sST2 tertile groups compared to tertile 1 . Inflammatory biomarkers, including high sensitivity C-reactive protein (hs-CRP), white blood cell (WBC) and eosinophil count, were not significantly different among three groups of sST2 concentration. There were no differences in the short- and long-term MACEs based on SST2 concentration.

\section{sST2 and echocardiographic angiographic data}

A summary of the echocardiographic data is provided in Tables 2 and 3. When categorized by sST2 concentration, there were no significant differences in the baseline and follow-up in terms of LVEF, LVIDd, E/E', LAVI, and WMSI among the sST2 tertile groups (Table 2). However, in terms of changes in echocardiographic parameters, a lower sST2 concentration was associated with $\triangle \mathrm{LVEF}$ (absolute percent point difference of LVEF at 6 month vs. baseline; tertile $1,7.3[-0.8,15.8]$, $\mathrm{p}=0.001$; tertile $2,1.3[-4.3,9.1], \mathrm{p}=0.319$; tertile $3,1.7[-8.1,10.1], \mathrm{p}=0.205)$ and $\Delta \mathrm{WMSI}$ (absolute numeric difference of WMSI at 6 month vs. baseline; tertile $1,-0.1[-0.2,0], \mathrm{p}=0.001$; tertile $2,-0.1[-0.2,0.1], \mathrm{p}=0.013$; tertile 3 , $0[-0.3,0], \mathrm{p}=0.055$; Table 3 and Fig. 2).

sST2 levels in relation to other biomarkers and risk stratification strategies

A significant univariate association was found only between baseline sST2 concentration and $\triangle$ LVEF $(r=-0.232, \mathrm{p}=0.018)$. The baseline troponin-T level was not statistically significant but showed a correlation tendency with baseline $\mathrm{sST} 2$ concentration $(\mathrm{r}=0.144, \mathrm{p}=0.051)$. Following adjustment for the relevant variables, partial correlation analysis showed a constant association between $\mathrm{SST} 2$ concentration and $\triangle \mathrm{LVEF}$ $\left(\mathrm{r}=-0.273, \mathrm{p}=0.006\right.$; adjusted by $\Delta \mathrm{LVIDd}, \Delta \mathrm{E} / \mathrm{E}^{\prime}$, $\triangle \mathrm{LAVI}$, and $\Delta \mathrm{WMSI})$.

According to categories in the known risk stratification strategies, including Killip classification, TIMI risk score, and CACS score, there were no significant differences between risk scores (Fig. 3). In the linear regression model, no significant associations were found between sST2 and known risk stratification strategies (sST2 and Killip classification, $\beta=0.005, \mathrm{p}=0.320$; sST2 and TIMI risk score, $\beta=0.008, \mathrm{p}=0.220$; $\mathrm{sST} 2$ and CACS score, $\beta=0.008, \mathrm{p}=0.222$ ). However, there were significant associations among risk stratification strategies (TIMI risk score and Killip classification, $\beta=0.382, \mathrm{p}<0.001 ;$ CACS score and Killip clas- 
Table 2. Echocardiographic parameters according to sST2 tertile in patients with ST-segment elevation myocardial infarction.

\begin{tabular}{|c|c|c|c|c|}
\hline \multirow[t]{2}{*}{ Variables } & \multicolumn{3}{|c|}{ sST2 [ng/mL] } & \multirow[t]{2}{*}{$\mathbf{P}$} \\
\hline & $\begin{array}{c}\text { Tertile } 1 \\
23.2 \leq \text { sST2 }<53.6\end{array}$ & $\begin{array}{c}\text { Tertile } 2 \\
53.6 \leq \mathrm{sST} 2<72.0\end{array}$ & $\begin{array}{c}\text { Tertile } 3 \\
72.0 \leq \mathrm{sST} 2<197.5\end{array}$ & \\
\hline \multicolumn{5}{|c|}{ Baseline $(n=181)$} \\
\hline LVEF [\%] & $58(46,66)$ & $62(56,69)$ & $58(52,66)$ & 0.241 \\
\hline LVIDd [mm] & $50(47,52)$ & $51(46,54)$ & $50(46,54)$ & 0.687 \\
\hline$E / E^{\prime}$ & $10.3(8.1,12.6)$ & $10.4(8.5,13.9)$ & $11.7(8.8,16.2)$ & 0.319 \\
\hline $\mathrm{LAVI}\left[\mathrm{mL} / \mathrm{m}^{2}\right]$ & $27.9(25.5,33.3)$ & $30.5(26.1,39.0)$ & $29.7(24.5,36.5)$ & 0.178 \\
\hline WMSI & $1.4(1.1,1.8)$ & $1.4(1.1,1.6)$ & $1.4(1.1,1.7)$ & 0.498 \\
\hline \multicolumn{5}{|c|}{ 6-month follow-up ( $n=103$ ) } \\
\hline LVEF (\%] & $63(56,70)$ & $64(58,72)$ & $61(53,69)$ & 0.676 \\
\hline LVIDd [mm] & $51(48,54)$ & $51(48,55)$ & $51(47,54)$ & 0.841 \\
\hline$E / E^{\prime}$ & $9.3(7.7,11.8)$ & $9.9(8.5,14.0)$ & $9.6(8.4,11.2)$ & 0.564 \\
\hline LAVI $\left[\mathrm{mL} / \mathrm{m}^{2}\right]$ & $28.3(25.3,32.6)$ & $31.0(26.9,35.7)$ & $29.2(24.6,36.8)$ & 0.459 \\
\hline WMSI & $1.2(1.0,1.6)$ & $1.2(1.0,1.4)$ & $1.3(1.0,1.7)$ & 0.714 \\
\hline
\end{tabular}

Data are presented as number (\%) and mean (standard deviation) or median (interquartile). Non-parametric continuous variables, which were evaluated by the Kolmogorov-Smimov method, were analyzed by the Kruskal-Wallis test. LVEF — left ventricular ejection fraction; LVIDd - left ventricular internal dimension, diastolic; LAVI — left atrial volume index; WMSI — wall motions score index

Table 3. Comparisons of serial changes in echocardiographic parameters after 6 months compared to baseline.

\begin{tabular}{lcc}
\hline & $\begin{array}{c}\Delta \text { } \text { month } \\
\text { - baseline }\end{array}$ & P \\
\hline Tertile 1 (n = 34) & & \\
LVEF [\%] & $7.3(-0.8,15.8)$ & 0.001 \\
LVIDd [mm] & $0(-1.3,3.5)$ & 0.309 \\
E/E' & $-0.7(-2.4,1.8)$ & 0.487 \\
LAVI [mL/m²] & $1.2(-4.9,7.1)$ & 0.260 \\
WMSI & $-0.1(-0.2,0)$ & 0.001 \\
Tertile 2 (n = 33) & & \\
LVEF [\%] & $1.3(-4.3,9.1)$ & 0.319 \\
LVIDd [mm] & $-0.5(-3.2,2.9)$ & 0.894 \\
E/E' & $0.5(-2.1,2.8)$ & 0.889 \\
LAVI [mL/m²] & $1.2(-5.9,5.6)$ & 0.407 \\
WMSI & $-0.1(-0.2,0.1)$ & 0.013 \\
Tertile 3 (n = 36) & & \\
LVEF [\%] & $1.7(-8.1,10.1)$ & 0.205 \\
LVIDd [mm] & $1.2(-1.2,3.8)$ & 0.067 \\
E/E' & $-1.0(-2.9,1.0)$ & 0.090 \\
LAVI [mL/m²] & $-0.6(-5.3,7.8)$ & 0.972 \\
WMSI & $0(-0.3,0)$ & 0.055 \\
\hline Data are presented as median (interquartile) and were analyzed \\
through Wilcoxon signed rank sum test. LVEF - left ventricular \\
ejection fraction; LVIDd - left ventricular internal dimension, dias- \\
tolic; LAVI - left atrial volume index; WMSI - wall motions score \\
index
\end{tabular}

sification, $\beta=0.605, \mathrm{p}<0.001$; TIMI risk score and CACS score, $\beta=0.658, \mathrm{p}<0.001$ ).

\section{Association between adverse outcomes and sST2 concentration}

Over the course of 1 year following the index PCI, 6 MACE occurred (6 cardiovascular deaths), with an event rate of $3 \%$, and all events occurred within 30 days. Cox regression analysis was used to identify independent predictors for MACE after primary PCI, and the results are shown in Table 4. Baseline systolic blood pressure, symptom to door time, symptom to balloon time, TIMI risk score, and CACS score were independently associated with 1-year MACE by univariate analysis. After adjusting these variables, baseline systolic blood pressure (HR 0.97 [0.94-0.99], $\mathrm{p}=0.011$ ) was found to independently predict 1-year MACE in this registry. sST2 concentration was not shown to be associated with both short- and long-term outcomes by survival analysis (Fig. 4).

\section{Discussion}

\section{Main findings}

The current study sought to explore the relationship among pre-procedural serum sST2 concentration and clinical, echocardiographic, and laboratory results in patients with STEMI. The 


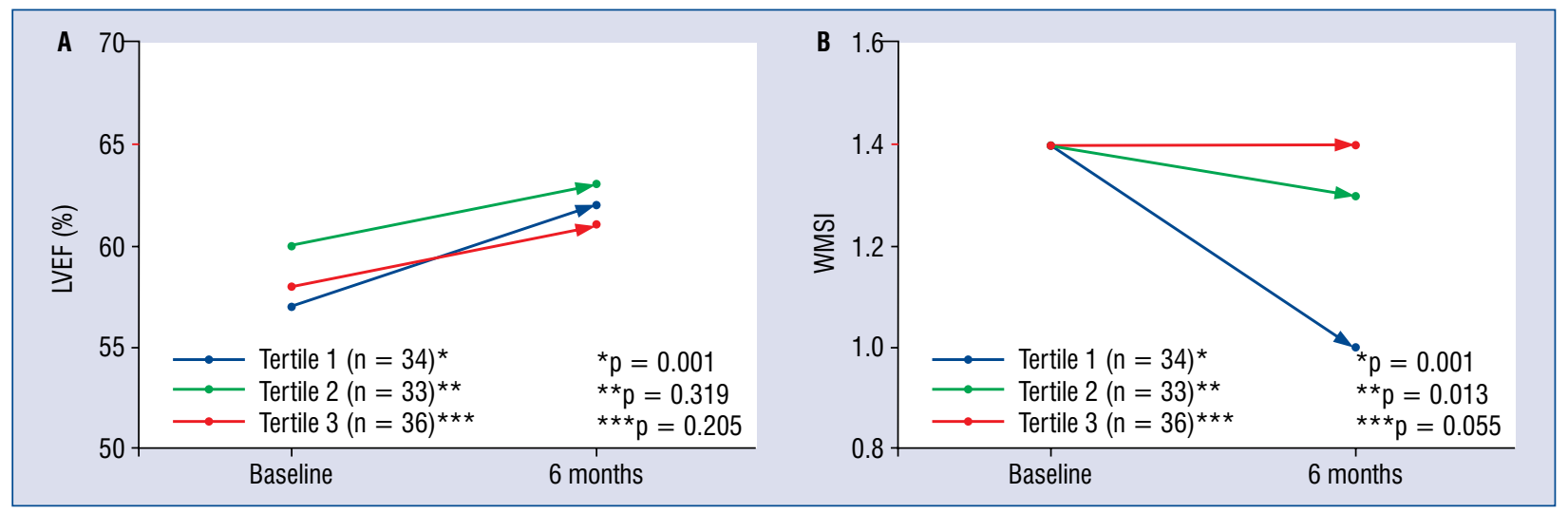

Figure 2. Time-dependent changes in left ventricular ejection fraction (LVEF; A) and wall motions score index (WMSI; B) by sST2 tertile groups.

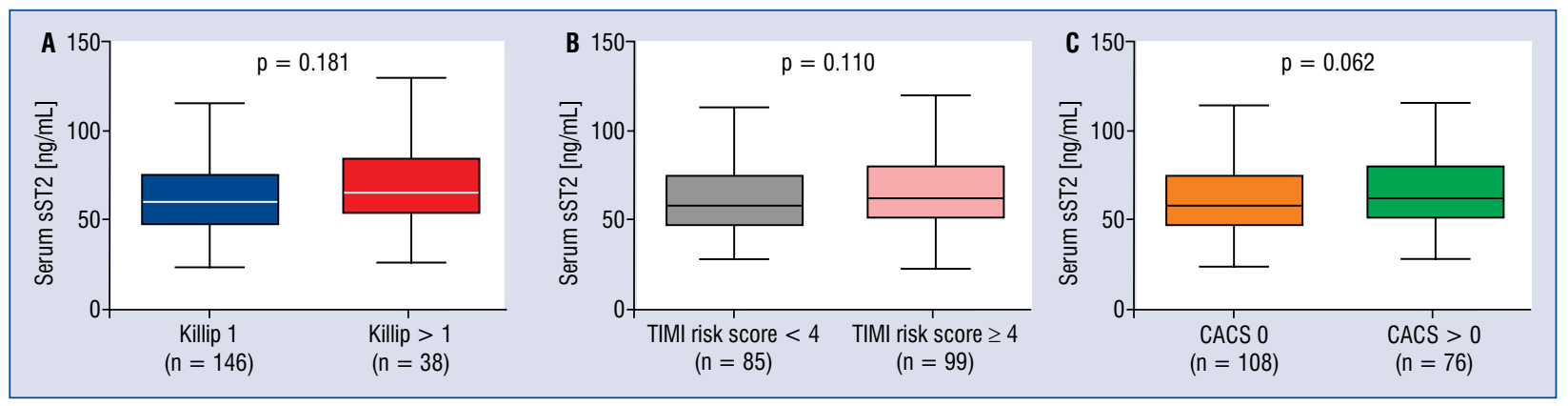

Figure 3. Comparison of sST2 level for Killip, TIMI risk score, CACS score classification in patients with ST-segment elevation myocardial infarction; A. Killip classification 1 vs. $>1$; B. TIMI risk score $<4$ vs. $\geq 4$; C. CACS score 0 vs. $>0$; $\mathrm{TIMI}$ - thrombolysis in myocardial infarction; CACS - Canadian acute coronary syndrome.

results demonstrated that an elevated concentration of sST2 was a negative predictor of improvement in LV systolic function 6 months after index primary PCI and lower sST2 tertile groups were associated with a significant improvement in WMSI at 6 months. However, a higher sST2 level was not shown to be a predictor of adverse cardiovascular outcomes, independent of traditional risk stratification strategies, including the TIMI risk score, Killip classification, and CACS score for STEMI. Furthermore, the sST2 level was not associated with other biomarkers, including peak CPK, CK-MB, and cardiac specific troponin, and was not shown to be associated with other risk stratification strategies. The location of culprit lesions was not associated with serum sST2 concentration. there was no statistical association found between the serum sST2 concentration and adverse cardiovascular outcomes after primary PCI in this single registry.

\section{sST2 and cardiovascular disease}

It is known that ST2, an interleukin-1 receptor family member, is basally expressed by cardiomyocytes [20]. ST2 consists of membrane and soluble forms, and an increase in soluble ST2 has been shown to negatively impact the cardioprotective effect, which in turn, can lead to myocardial remodeling and fibrosis [21, 22]. This finding raised the possibility that the concentration of sST2 may be of predictive value in cardiovascular disease. Indeed, preclinical studies have shown upregulation of sST2 in cardiomyocytes in models of MI [23], while clinical studies have demonstrated the association between a higher sST2 concentration and adverse cardiovascular outcomes in patients with STEMI [13-16, 24]. Furthermore, several studies have demonstrated that short-term changes in sST2 concentration were prognostic of mortality in severe HF [25] among dyspneic patients with 
Table 4. Cox regression analysis for predictors of 1-year major adverse cardiac events.

\begin{tabular}{|c|c|c|c|c|}
\hline & \multicolumn{2}{|c|}{ Univariable analysis } & \multicolumn{2}{|c|}{ Multivariable model $1 *$} \\
\hline & Unadjusted HR (95\% Cl) & $\mathbf{P}$ & Adjusted HR (95\% CI) & $\mathbf{P}$ \\
\hline Age & $1.03(0.96-1.11)$ & 0.364 & & \\
\hline Female & $2.27(0.50-14.9)$ & 0.247 & & \\
\hline Smoking & $0.45(0.09-2.23)$ & 0.328 & & \\
\hline Hypertension & $4.26(0.50-36.4)$ & 0.186 & & \\
\hline Diabetes & $1.09(0.20-5.92)$ & 0.925 & & \\
\hline Baseline SBP & $0.97(0.95-0.99)$ & 0.001 & $0.97(0.94-0.99)$ & 0.011 \\
\hline Symptom to door time & $1.00(1.00-1.00)$ & 0.035 & $1.00(1.00-1.00)$ & 0.022 \\
\hline Symptom to balloon time & $1.00(1.00-1.00)$ & 0.036 & & \\
\hline Door to balloon time & $1.00(0.95-1.06)$ & 0.974 & & \\
\hline LAD vs. non-LAD lesion & $1.53(0.28-8.34)$ & 0.624 & & \\
\hline Killip classification & $1.61(0.90-2.89)$ & 0.111 & & \\
\hline TIMI risk score & $1.56(1.12-2.16)$ & 0.009 & $1.27(0.68-2.37)$ & 0.451 \\
\hline CACS score & $2.73(1.27-5.89)$ & 0.010 & $0.85(0.16-4.36)$ & 0.840 \\
\hline Peak CPK & $1.00(1.00-1.00)$ & 0.351 & & \\
\hline Peak CK-MB & $1.00(0.99-1.01)$ & 0.978 & & \\
\hline Peak troponin $\mathrm{T}$ & $1.30(1.02-1.65)$ & 0.170 & & \\
\hline sST2 & $0.99(0.95-1.02)$ & 0.439 & & \\
\hline \multicolumn{5}{|l|}{ Tertile by sST2: } \\
\hline Tertile 2 vs. 1 & $1.53(0.26-9.12)$ & 0.644 & & \\
\hline Tertile 3 vs. 1 & $0.51(0.05-5.60)$ & 0.580 & & \\
\hline
\end{tabular}

* Model 1: Adjusted for the baseline SBP, symptom to door time, TIMI risk score, and CACS score. The pro-BNP was not included in the analysis due to the small number of subjects. $\mathrm{Cl}$ - confidence interval, HR - hazard ratio; rest abbreviations are defined in Tables 1 and 2.

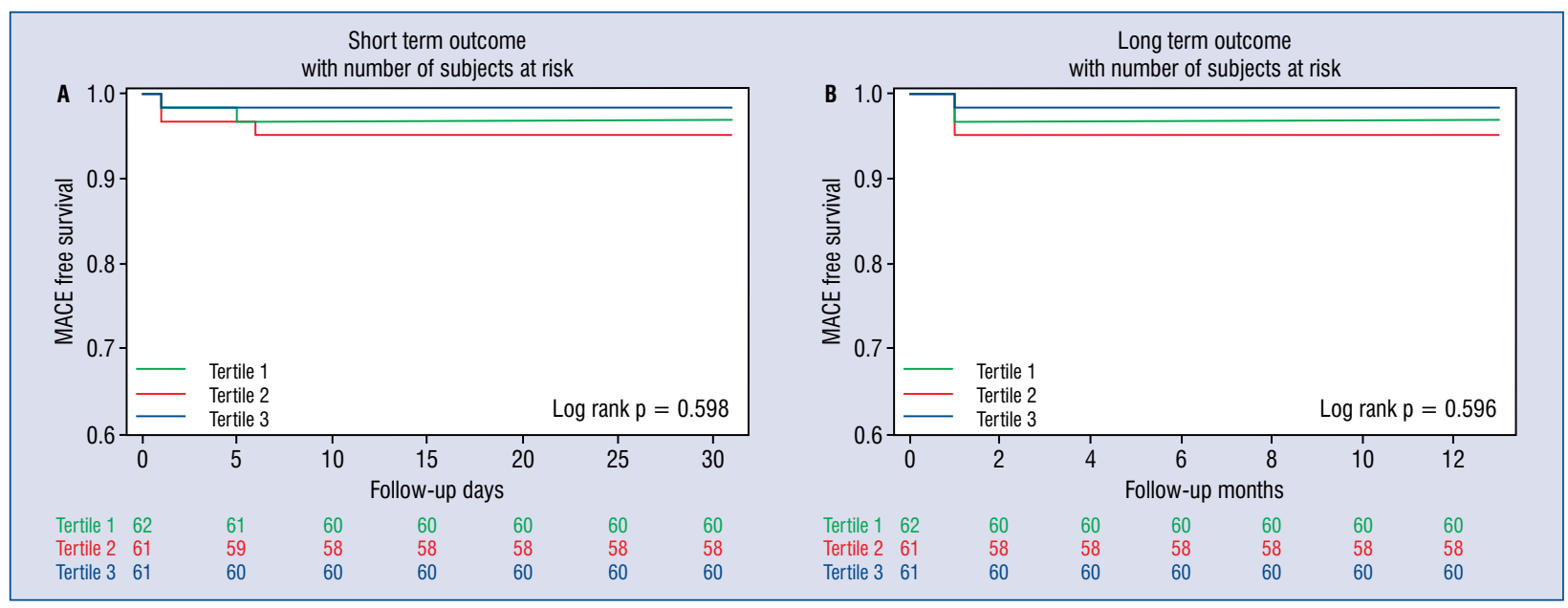

Figure 4. Cumulative incidence of major cardiac adverse events (MACE) during 30 days (A) and 1-year (B) by sST2 tertile in patients with ST-segment elevation myocardial infarction.

and without acute HF [26]. Many further reports corroborated the prognostic power of sST2 in multiple acute and chronic cardiovascular settings $[27,28]$.
SST2 as a predictor of 30-day and 1-year MACE after primary PCI

Two reports on data derived from three randomized clinical trials in patients with STEMI 
provide data on the predictive value of serum sST2 concentration for adverse outcome up to 30-days after MI, while further studies reported on prognostic implications up to a median follow-up period of 20 months [13, 29, 30]. Shimpo et al. [29] showed that an ascending quartile of serum SST2 concentration significantly corresponded to increasing time from symptom onset, higher heart rates, higher cardiac troponin-I, higher B-type natriuretic peptide (BNP), higher CRP, higher creatinine, and an increasing likelihood of an anterior location of the MI. However, in the present study, the sST2 level was not correlated with other biomarkers, culprit lesion of MI, and time from symptom onset to door/balloon. Sabatine et al. [13] revealed that sST2 and NT-proBNP were found to have complementary roles in STEMI compared to the TIMI risk score. Dhillon et al. [30] also demonstrated a correlation between sST2 and the Global Registry of Acute Coronary Events (GRACE) risk score. However, in the current study, the proBNP level was collected in only 19 subjects and performing a correlation analysis between sST2 and proBNP was not possible. Furthermore, sST2 concentration was not associated with risk stratification strategies including TIMI risk score, Killip classification, and CACS score.

Although a small number of subjects have been included, contrary to prior studies in STEMI $[13,15,16]$, the present results did not provide a prognostic power of serum sST2 concentration for adverse cardiovascular outcomes. One possible argument for this discrepancy is that restoration time of flow from symptoms onset affect to myocardial damage which is related to increased biomechanical strain that causes higher sST2 levels. Severe myocardial damage and remodeling is expected in a relatively long term from symptom onset. Previous studies have revealed the time from symptom onset to lytic therapy $2.4 \pm 1.3 \mathrm{~h}$ to $4.2 \pm 3.0 \mathrm{~h}$ [13], and $2.8 \pm 1.6$ to $4.0 \pm 1.9$ [29]. Analysis of serial measurements of serum sST2 in 228 patients showed an increase sST2 with time especially after $3 \mathrm{~h}$, with a peak level at $12 \mathrm{~h}$ for most patients [29]. It was identified herein, that the time from symptom to PCI (median; $2.7-2.8 \mathrm{~h}$ ) was revealed to be relatively less than in previous studies. This indicates that, perhaps the impact of serum sST2 level would not have been strong in this study.

\section{sST2 and LV remodeling}

While data related to circulating sST2 concentration to cardiac function and structure are variable and sparse, some reports have shown a weak inverse relationship between sST2 level and various cardiovascular disease cohorts [31]. Weir et al. [32] analyzed the relationship between sST2 and serial change in LV function after acute MI measured by cardiac magnetic resonance imaging, NT-proBNP, norepinephrine, and aldosterone at baseline and at 12 - and 24-week follow-up. It was demonstrated that sST2 had a significant inverse correlation with the change in LVEF between baseline and 6-month follow-up. In addition, the LV enddiastolic volume index was correlated with changes in SST2 concentration. An inverse correlation was demonstrated between the serial change in LVEF, WMSI by transthoracic echocardiography, and baseline sST2 tertile. Furthermore, the LVEF was significantly increased after 6 months in tertile 1 , and WMSI was significantly improved after 6 months in tertiles 1 and 2 . The serum sST2 concentration after STEMI was related to mid-term changes in LV function and remodeling.

\section{Limitations of the study}

The present study should be interpreted in the context of its limitations. First, the present study is observational and was a relatively small single-center retrospective study. The treatment groups may have been confounded by selection bias. Nevertheless, the cohort registry was homogenous, and all study populations included STEMI patients who underwent primary PCI and were managed using the same protocol. Secondly, the blood for sST2 measurements was taken at the presentation of STEMI, and the follow-up sST2 values were not examined. Third, $93 \%, 86 \%$, and $81 \%$ of patients not $100 \%$ took statin, beta-blocker, and angiotensin converting enzyme inhibitor (ACEI) or angiotensin II receptor blocker (ARB) during in-hospital day due to elevated liver enzyme in the case of statin, marked sinus bradycardia even if there were no symptoms in the case of beta-blocker, and suspected acute kidney injury or electrolyte imbalance, such as hyperkalemia in the case of ACEI or ARB. However, most of these drugs were administered unless there was a specific contraindication during outpatient clinic term. Fourth, the time from the first symptom onset to hospital or PCI was quite short compared to that of the Korea Acute Myocardial Infarction Registry (KAMIR), which is the nationwide, prospective, multicenter registry of Korean patients with acute MI (symptom onset to balloon time; median $220 \mathrm{~min}$ at 2014; $210 \mathrm{~min}$ at $2015 ; 200 \mathrm{~min}$ at 2016 ; and $212 \mathrm{~min}$ at 2017) [3]. Differences were found, including short-and long-term MACE, in this registry compared to 
the KAMIR data. Although it is considered possible that a relatively short reperfusion time from symptom onset may have affected the outcome, this could not be determined in this study.

Finally, most previous studies of sST2 in cardiac disease applied different assays than those used in the current study; this limits the transferability of the present results to findings of previous investigations.

\section{Conclusions}

In conclusion, lower values of sST2, obtained at the time of presentation at hospital in patients with STEMI resulted in less damaged myocardium and improved LV systolic function in the mid-term which is associated with a lesser likelihood of LV remodeling. However, higher values of sST2 were not associated with either short- or long-term MACE. Data herein, provides valuable information on clinical outcomes and the structural association with sST2 concentration.

\section{Conflict of interest: None declared}

\section{References}

1. Mozaffarian D, Benjamin EJ, Go AS, et al. Heart Disease and Stroke Statistics-2016 Update: A Report From the American Heart Association. Circulation. 2016; 133(4): e38-360, doi: 10.1161/CIR.0000000000000350, indexed in Pubmed: 26673558.

2. Ibanez B, James S, Agewall S, et al. ESC Scientific Document Group. 2017 ESC Guidelines for the management of acute myocardial infarction in patients presenting with ST-segment elevation: The Task Force for the management of acute myocardial infarction in patients presenting with ST-segment elevation of the European Society of Cardiology (ESC). Eur Heart J. 2018; 39(2): 119-177, doi: 10.1093/eurheartj/ehx393, indexed in Pubmed: 28886621.

3. Kim Y, Ahn Y, Cho MC, et al. Current status of acute myocardial infarction in Korea. Korean J Intern Med. 2019; 34(1): 1-10, doi: 10.3904/kjim.2018.381, indexed in Pubmed: 30612415.

4. Sim DS, Jeong MHo. Differences in the Korea Acute Myocardial Infarction Registry Compared with Western Registries. Korean Circ J. 2017; 47(6): 811-822, doi: 10.4070/kcj.2017.0027, indexed in Pubmed: 29035427.

5. Menees DS, Peterson ED, Wang Y, et al. Door-to-balloon time and mortality among patients undergoing primary PCI. N Engl J Med. 2013; 369(10): 901-909, doi: 10.1056/NEJMoa1208200, indexed in Pubmed: 24004117.

6. Afilalo J, Piazza N, Tremblay S, et al. Symptom-to-door time in ST segment elevation myocardial infarction: overemphasized or overlooked? Results from the AMI-McGill study. Can J Cardiol. 2008; 24(3): 213-216, doi: 10.1016/s0828-282x(08)70587-7, indexed in Pubmed: 18340392.

7. Lindahl B, Toss H, Siegbahn A, et al. Markers of myocardial damage and inflammation in relation to long-term mortality in unstable coronary artery disease. FRISC Study Group. Fragmin during Instability in Coronary Artery Disease. N Engl J Med. 2000; 343(16): 1139-1147, doi: 10.1056/NEJM200010193431602, indexed in Pubmed: 11036119.

8. Chan D, Ng LL. Biomarkers in acute myocardial infarction. BMC Med. 2010; 8: 34, doi: 10.1186/1741-7015-8-34, indexed in Pubmed: 20529285

9. Vieira C, Nabais S, Ramos V, et al. Multimarker approach with cystatin $\mathrm{C}, \mathrm{N}$-terminal pro-brain natriuretic peptide, C-reactive protein and red blood cell distribution width in risk stratification of patients with acute coronary syndromes. Rev Port Cardiol. 2014; 33: 127-36.

10. Sanada S, Hakuno D, Higgins LJ, et al. IL-33 and ST2 comprise a critical biomechanically induced and cardioprotective signaling system. J Clin Invest. 2007; 117(6): 1538-1549, doi: 10.1172/ JCI30634, indexed in Pubmed: 17492053.

11. Turnquist HR, Zhao $Z$, Rosborough BR, et al. IL-33 expands suppressive $\mathrm{CD} 11 \mathrm{~b}+\mathrm{Gr}-1$ (int) and regulatory $\mathrm{T}$ cells, including ST2L + Foxp3+ cells, and mediates regulatory T cell-dependent promotion of cardiac allograft survival. J Immunol. 2011; 187(9): 4598-4610, doi: 10.4049/jimmunol.1100519, indexed in Pubmed: 21949025.

12. Seki K, Sanada S, Kudinova AY, et al. Interleukin-33 prevents apoptosis and improves survival after experimental myocardial infarction through ST2 signaling. Circ Heart Fail. 2009; 2(6): 684-691, doi: 10.1161/CIRCHEARTFAILURE.109.873240, indexed in Pubmed: 19919994.

13. Sabatine MS, Morrow DA, Higgins LJ, et al. Complementary roles for biomarkers of biomechanical strain ST2 and N-terminal prohormone B-type natriuretic peptide in patients with ST-elevation myocardial infarction. Circulation. 2008; 117(15): 1936-1944, doi: 10.1161/CIRCULATIONAHA.107.728022, indexed in Pubmed: 18378613.

14. Kohli P, Bonaca MP, Kakkar R, et al. Role of ST2 in non-ST-elevation acute coronary syndrome in the MERLIN-TIMI 36 trial. Clin Chem. 2012; 58(1): 257-266, doi: 10.1373/clinchem.2011.173369, indexed in Pubmed: 22096031.

15. Yu J, Oh PC, Kim M, et al. Improved early risk stratification of patients with ST-segment elevation myocardial infarction undergoing primary percutaneous coronary intervention using a combination of serum soluble ST2 and NT-proBNP. PLoS One. 2017; 12(8): e0182829, doi: 10.1371/journal.pone.0182829, indexed in Pubmed: 28796845.

16. Jenkins WS, Roger VL, Jaffe AS, et al. Prognostic Value of Soluble ST2 After Myocardial Infarction: A Community Perspective. Am J Med. 2017; 130(9): 1112.e9-1112.e15, doi: 10.1016/j.amjmed.2017.02.034, indexed in Pubmed: 28344136.

17. Kuroiwa K, Li H, Tago K, et al. Construction of ELISA system to quantify human ST2 protein in sera of patients. Hybridoma. 2000; 19(2): 151-159, doi: 10.1089/02724570050031194, indexed in Pubmed: 10868795.

18. Dieplinger B, Januzzi JL, Steinmair M, et al. Analytical and clinical evaluation of a novel high-sensitivity assay for measurement of soluble ST2 in human plasma--the Presage ST2 assay. Clin Chim Acta. 2009; 409(1-2): 33-40, doi: 10.1016/j. cca.2009.08.010, indexed in Pubmed: 19699192.

19. Mitchell C, Rahko PS, Blauwet LA, et al. Guidelines for Performing a Comprehensive Transthoracic Echocardiographic Examination in Adults: Recommendations from the American Society of Echocardiography. J Am Soc Echocardiogr. 2019; 32(1): 1-64, doi: 10.1016/j.echo.2018.06.004, indexed in Pubmed: 30282592. 
20. Weinberg E, Shimpo M, Keulenaer GDe, et al. Expression and Regulation of ST2, an Interleukin-1 Receptor Family Member, in Cardiomyocytes and Myocardial Infarction. Circulation. 2002; 106(23): 2961-2966, doi: 10.1161/01.cir.0000038705.69871.d9.

21. Pascual-Figal DA, Januzzi JL. The biology of ST2: the International ST2 Consensus Panel. Am J Cardiol. 2015; 115(7 Suppl): 3B-7B, doi: 10.1016/j.amjcard.2015.01.034, indexed in Pubmed: 25665766.

22. Vasile VC, Jaffe AS. Emerging biomarkers for acute heart conditions. Curr Opin Cardiol. 2014; 29(4): 312-318, doi: 10.1097/ HCO.0000000000000077, indexed in Pubmed: 24806784.

23. Kakkar R, Lee RT. The IL-33/ST2 pathway: therapeutic target and novel biomarker. Nat Rev Drug Discov. 2008; 7(10): 827-840, doi: 10.1038/nrd2660, indexed in Pubmed: 18827826.

24. Eggers KM, Armstrong PW, Califf RM, et al. ST2 and mortality in non-ST-segment elevation acute coronary syndrome. Am Heart J. 2010; 159(5): 788-794, doi: 10.1016/j.ahj.2010.02.022, indexed in Pubmed: 20435187.

25. Weinberg EO, Shimpo M, Hurwitz S, et al. Identification of serum soluble ST2 receptor as a novel heart failure biomarker. Circulation. 2003; 107(5): 721-726, doi: 10.1161/01. cir.0000047274.66749.fe, indexed in Pubmed: 12578875.

26. Januzzi JL, Peacock WF, Maisel AS, et al. Measurement of the in terleukin family member ST2 in patients with acute dyspnea: results from the PRIDE (Pro-Brain Natriuretic Peptide Investigation of Dyspnea in the Emergency Department) study. J Am Coll Cardiol. 2007; 50(7): 607-613, doi: 10.1016/j.jacc.2007.05.014, indexed in Pubmed: 17692745.
27. Richards AM, Di Somma S, Mueller T. ST2 in stable and unstable ischemic heart diseases. Am J Cardiol. 2015; 115(7 Suppl): 48B-58B, doi: 10.1016/j.amjcard.2015.01.041, indexed in Pubmed: 25678392.

28. Januzzi JL, Pascual-Figal D, Daniels LB. ST2 testing for chronic heart failure therapy monitoring: the International ST2 Consensus Panel. Am J Cardiol. 2015; 115(7 Suppl): 70B-75B, doi: 10.1016/j.amjcard.2015.01.044, indexed in Pubmed: 25670638.

29. Shimpo M, Morrow DA, Weinberg EO, et al. Serum levels of the interleukin-1 receptor family member ST2 predict mortality and clinical outcome in acute myocardial infarction. Circulation. 2004; 109(18): 2186-2190, doi: 10.1161/01.CIR.0000127958.21003.5A, indexed in Pubmed: 15117853.

30. Dhillon OS, Narayan HK, Khan SQ, et al. Pre-discharge risk stratification in unselected STEMI: is there a role for ST2 or its natural ligand IL-33 when compared with contemporary risk markers? Int J Cardiol. 2013; 167(5): 2182-2188, doi: 10.1016/j. ijcard.2012.05.073, indexed in Pubmed: 22835988.

31. Okar S, Kaypakli O, Şahin DY, et al. Fibrosis Marker Soluble ST2 Predicts Atrial Fibrillation Recurrence after Cryoballoon Catheter Ablation of Nonvalvular Paroxysmal Atrial Fibrillation. Korean Circ J. 2018; 48(10): 920-929, doi: 10.4070/kcj.2018.0047, indexed in Pubmed: 30238709.

32. Weir RAP, Miller AM, Murphy GEJ, et al. Serum soluble ST2: a potential novel mediator in left ventricular and infarct remodeling after acute myocardial infarction. J Am Coll Cardiol. 2010; 55(3): 243-250, doi: 10.1016/j.jacc.2009.08.047, indexed in Pubmed: 20117403. 